\title{
Electrohydrodynamic Interfacial Stability Conditions in the Presence of Heat and Mass Transfer and Oblique Electric Fields
}

\author{
Mohamed Fahmy El-Sayed \\ Department of Mathematics and Computer Science, Faculty of Science, Unit Arab Emirates \\ University, P.O. Box 17551 Al Ain, U.A.E. \\ also at: Department of Mathematics, Faculty of Education, Ain Shams \\ University, Roxy, Cairo, Egypt. \\ Reprint requests to Dr. M. F. El-Sayed; E-mail address: elsayed@nyx.uaeu.ac.ae; Fax: (9713) 671291. \\ Z. Naturforsch. 54a, 470-476 (1999); received June 9, 1999

\begin{abstract}
A novel mathematical formulation to deal with interfacial stability problems of the Kelvin-Helmholtz type with heat and mass transfer in the presence of oblique electric fields is presented. The perturbed system is composed of two homogeneous, inviscid, incompressible, dielectric, and streaming fluids separated by a horizontal interface, and bounded by two rigid planes. The effect of a phase transition on the instability is considered, and the linear dispersion relations are obtained and discussed. It is found that the electric field has a major effect and can be chosen to stabilize or destabilize the flow. For Rayleigh-Taylor instability problems of a liquid-vapor system it is found that the effect of mass and heat transfer enhances the stability of the system when the vapor is hotter than the liquid, although the classical stability criterion is still valid. For Kelvin-Helmholtz instability problems, however, the classical stability criterion is found to be substantially modified due to the effects of the electric field, mass and heat transfer. A new stability condition relating the magnitude and orientation of the electric field and the dielectric constants is obtained. Oblique electric fields are found to have stabilizing effects which are reduced by the normal components of the electric fields. The effects of orientation of the electric fields and fluid depths on the stability configuration are also discussed.
\end{abstract}

PACS: 47.20.-k; 47.27.Tc; 47.65.+a.

Key words: Hydrodynamic Stability; Convection and Heat Transfer; Electrohydrodynamics.

\section{Introduction}

The problem of the interfacial stability of the flow of two immiscible fluids separated by an interface is usually studied when there is no mass transfer across the interface [1]. Thermal effects often play only a secondary role; therefore the effect of heat transfer is also usually neglected. However there are situations when the effect of heat and mass transfer across the interface plays an essential role in determining the flow field. For example, when the fluid is boiling, whether film boiling or pool boiling, the motion of the film and the bubbles depends essentially on the effect of heat and mass transfer.

The hydrodynamic stability of two phase systems has long been studied for a better understanding of numerous physically significant situations. One case of interest that includes both the Rayleigh-Taylor and KelvinHelmholtz instabilities, is film boiling heat transfer. Hsieh [2] developed a mathematical formulation for a liquid-vapor interface with heat and mass transfer and derived the dispersion relations for the Rayleigh-Taylor and Kelvin-Helmholtz instabilities for inviscid vapor and liquid. Nayak and Chakraborty [3] extended the KelvinHelmholtz analysis to the case of cylindrical interfaces of nonviscous fluids. Ho [4] considered the linear stability of stationary, viscous fluids with interfacial heat and mass transfer and derived a new dispersion relation which showed a critical wavenumber differing from the classical one. For recent works on linear stability problems with heat and mass transfer, see [5-9].

Moreover, for the specific problem of boiling heat transfer, it is evident that a study of the nonlinear problems is required in order to really understand its physical mechanism. Hsieh [10] used the multiple expansion method to study the nonlinear Rayleigh-Taylor instability of a liquid layer over a finite vapor layer under the assumption that the flows are irrotational. It is observed that the nonlinear effects can indeed increase the range of stability of the system when there is strong heat and mass transfer. In the nonlinear analysis, the size of the vapor bubbles which detach from the interface can also be estimated. Chhabra and Trehan [11] examined this problem in the more general case when no restriction is made on the nature of the flow, i.e. when it is not neces- 
sarily restricted to be irrotational. They found that the region of stability increases, and the size of the bubbles which detach from the interface will be small compared to the ones obtained under restricted flows. For recent works on nonlinear stability physical problems of interest in the presence of heat and mass transfer, see [12-16].

The interaction of an electric field with a fluid is the subject of a branch of fluid mechanics called electrohydrodynamics. The literature on this subject concerned mainly surface phenomena such as charge relaxations and surface waves [17]. Through all regimes of electrohydrodynamic (EHD) coupled heat transfer in liquids has been studied; EHD enhanced film boiling seems to have received little attention in past experimental and theoretical work. It is known that, in the absence of an electric field, no convection is produced, since the system is gravitationally stable, but when the electric field strength is sufficiently large, then convection patterns are established [18]. A theory which predicts the effect of EHD forces on the minimum film boiling point is developed. This theory is concerning minimum film boiling, but modified to account for electrohydrodynamic coupling at the vapor-liquid interface. Experimental studies of electrohydrodynamic coupled film boiling have been untertaken by Choi [19], Bonjour et al. [20], Johnson [21], and Markels and Durfee [22]. All the aforementioned experimentalists have found that the minimum film boiling heat transfer is increased by strong electric fields, as shown theoretically by Jones and Schaeffer [23].

From a geophysical standpoint, the problem of the onset of convective instability in dielectric fluids under the simultaneous action of an applied electric field and a temperature gradient is of practical interest. Therefore in this work we present a simplified mathematical formulation of the problems of interfacial flow of the KelvinHelmholtz type to study the necessary stability conditions of a perturbed system composed of two non-viscous, incompressible, homogeneous, dielectric, and streaming fluids separated by a horizontal interface under the influence of oblique electric fields. The transfer of mass and heat across the interface is taken into account. Linear dispersion relations are obtained and discussed for both Rayleigh-Taylor and Kelvin-Helmholtz instabilities, in the presence of oblique electric fields.

\section{Mathematical Formulation of the Problem}

We consider the motion of two superposed homogeneous incompressible dielectric and inviscid fluids, bounded by two parallel conducting planes $y=-h_{1}$ and $y=h_{2}$ in the presence of heat and mass transfer. The fluid of density $\rho^{(1)}$, dielectric constant $\varepsilon^{(1)}$ occupies the region $-h_{1}<y<0$, and the fluid of density $\rho^{(2)}$, dielectric constant $\varepsilon^{(2)}$ occupies the region $0<y<h_{2}$, where the superscripts 1 and 2 refer to the lower and upper fluids, respectively, and $y=0$ is the equilibrium interface between the two fluids. The basic unperturbed flows have constant velocities $U^{(1)}$ and $U^{(2)}$ (in the $x$-direction).

Let the temperatures at $y=-h_{1}, y=h_{2}$, and $y=0$ be $T_{1}, T_{2}$, and $T_{0}$, respectively. The fluids are influenced by oblique electric fields at the interface between the two media, given by

$$
\boldsymbol{E}_{0}^{(j)}=E_{0}^{(j)}\left(\cos \theta_{j} \boldsymbol{e}_{x}+\sin \theta_{j} \boldsymbol{e}_{y}\right), \quad j=1,2,
$$

where $\boldsymbol{e}_{x}$ and $\boldsymbol{e}_{y}$ are unit vectors in the $x$-and $y$-directions, respectively, and $\theta_{j}$ are the angles between the fields and the $x$-axis. We shall assume that there are no volume charges present in the bulk of the fluids, and also there are no surface charges present at the interface in the equilibrium state, and will therefore vanish during the perturbations. The effect of viscosity will be neglected, while the effect of gravity $g$ is taken in the negative $y$-direction.

We assume that the quasi-static approximation is valid for this problem. Then the electric field can be derived from a scalar potential $\Psi$ (such that $E=-\nabla \Psi)$. The equations governing the motion, in each of the fluids are $[24,25]$

$$
\begin{aligned}
\frac{\partial v}{\partial t}+(\boldsymbol{v} \cdot \nabla) \boldsymbol{v} & =-\frac{1}{\rho} \nabla \Pi-g \boldsymbol{e}_{y}, \\
\nabla \cdot \boldsymbol{v} & =0 \\
\nabla \cdot(\varepsilon \boldsymbol{E}) & =0 \\
\nabla \times \boldsymbol{E}) & =0
\end{aligned}
$$

where $v$ is the fluid velocity, and $\Pi=p-(\varepsilon / 2) E^{2}$ is the modified pressure, $p$ being the hydrostatic pressure.

Consider the effect of small wave disturbations to the interface $y=0$, propagating in the positive $x$-direction. The surface deflection is assumed to be of the form

$$
S(x, y, t)=y-\xi(x, t)=y-\eta \exp i(k x-\omega t)=0,
$$

where $\eta$ is the amplitude, $k$ is the wavenumber of the interfacial wave, and $\omega$ is a constant. Assume that the motion of the fluids is irrotational, and let the velocity potentials be $\Phi^{(1)}$ and $\Phi^{(2)}$, respectively. For a small 
departure from the equilibrium state, the quantities $\boldsymbol{E}, \boldsymbol{v}$, $\Pi$, and $\Psi$ will receive increments $\boldsymbol{E}_{1}, \boldsymbol{v}_{1}, \Pi_{1}$, and $\Psi_{1}$, respectively, to yield

$$
\begin{aligned}
& \boldsymbol{E}^{(j)}=\boldsymbol{E}_{0}^{(j)}+\boldsymbol{E}_{1}^{(j)}, \boldsymbol{v}^{(j)}=U^{(j)} \boldsymbol{e}_{x}+\boldsymbol{v}_{1}^{(j)}, \\
& \Pi^{(j)}=\Pi_{0}^{(j)}+\Pi_{1}^{(j)}
\end{aligned}
$$

and $\Psi^{(j)}=\Psi_{0}^{(j)}+\Psi_{1}^{(j)}$,

where the subscript 0 refers to quantities in the equilibrium state.

The linearization of (2)-(5) leads to the equations

$$
\begin{aligned}
& \rho^{(j)}\left[\frac{\partial \boldsymbol{v}_{1}^{(j)}}{\partial t}+U^{(j)} \frac{\partial \boldsymbol{v}_{1}^{(j)}}{\partial x}\right]=-\nabla \Pi_{1}^{(j)}, \\
& \nabla \cdot \boldsymbol{v}_{1}^{(j)}=0, \text { where } \boldsymbol{v}_{1}^{(j)}=\nabla \boldsymbol{\Phi}^{(j)}, \\
& \nabla \cdot\left(\varepsilon^{(j)} \boldsymbol{E}_{1}^{(j)}\right)=0 \quad \text { and } \nabla \times \boldsymbol{E}_{1}^{(j)}=0 .
\end{aligned}
$$

Assuming the space and time dependence of the perturbed quantities to be of the form

$$
\text { זs }(x, y, t)=f(y) \exp i(k x-\omega t)
$$

then (9)-(11) lead to

$$
\begin{aligned}
& \nabla^{2} \Phi^{(j)}=0, \quad j=1,2, \\
& \nabla^{2} \Psi_{1}^{(j)}=0 \quad \text { and } \boldsymbol{E}_{1}^{(j)}=-\nabla \Psi_{1}^{(j)},
\end{aligned}
$$

where $j=1,2$ refer to the regions $-h_{1}<y<0$, and $0<y<h_{2}$, respectively.

\section{Boundary Conditions and Solutions}

The solutions of (12) and (13) can be written in the form

$$
\begin{aligned}
& \Psi_{1}^{(j)}=\frac{A_{j} \sinh k\left(y \pm h_{j}\right)}{\cosh k h_{j}} \exp i(k x-\omega t), \\
& \Phi^{(j)}= \pm \frac{C_{j} \cosh k\left(y \pm h_{j}\right)}{\sinh k h_{j}} \exp i(k x-\omega t),
\end{aligned}
$$

where $\Psi_{1}^{(j)}$ and $\Phi^{(j)}, j=1,2$, satisfy the boundary conditions

$$
\frac{\partial \Psi_{1}^{(j)}}{\partial x}=\frac{\partial \Phi^{(j)}}{\partial y}=0 \quad \text { at } y=\mp h_{j},
$$

To determine the constants $A_{j}$ and $C_{j}, j=1,2$, we use the following boundary conditions at the interface $y=\xi(x, t)$ :
(1) The conservation of mass across the interface is

$$
\begin{aligned}
\rho^{(1)} & {\left[\frac{\partial \Phi^{(1)}}{\partial y}-\left(\frac{\partial}{\partial t}+U^{(1)} \frac{\partial}{\partial x}\right) \xi\right] } \\
& =\rho^{(2)}\left[\frac{\partial \Phi^{(2)}}{\partial y}-\left(\frac{\partial}{\partial t}+U^{(2)} \frac{\partial}{\partial x}\right) \xi\right] .
\end{aligned}
$$

(2) The interfacial condition for energy is expressed as [2]

$$
L \rho^{(1)}\left[\frac{\partial \Phi^{(1)}}{\partial y}-\left(\frac{\partial}{\partial t}+U^{(1)} \frac{\partial}{\partial x}\right) \xi\right]=F(\xi),
$$

where $L$ is the latent heat released when the fluid is transformed from phase 1 to phase 2 . The expression $F(\xi)$ essentially represents the net heat flux from the interface when such a phase transaction is taking place. In general, the heat fluxes have to be determined from equations governing the heat transfer in the fluids, thus completely coupling the dynamics and the thermal exchanges in the entire flow region. In our simplified problem, the assumption is that $F$ is simply a function of $\xi$, and moreover $F$ is to be determined from the heat exchanges relations in the equilibrium state.

In our specific equilibrium problem, the heat fluxes in the $y$ direction in regions 1 and 2 are $K^{(1)}\left(T_{1}-T_{0}\right) / h_{1}$ and $K^{(2)}\left(T_{0}-T_{2}\right) / h_{2}$, where $K^{(1)}$ and $K^{(2)}$ denote the lower and upper thermal conductivities, respectively. Let us denote

$$
F(y)=\frac{K^{(2)}\left(T_{0}-T_{2}\right)}{h_{2}-y}-\frac{K^{(1)}\left(T_{1}-T_{0}\right)}{h_{1}+y} .
$$

It is clear that $F(0)$ represents the net heat flux from the interface into the fluid regions. Since it is an equilibrium state, we have $F(0)=0$. Now, we can expand $F(\xi)$ about $\xi=0$ by

$$
F(\xi)=F^{\prime}(0) \xi+\frac{F^{\prime \prime}(0)}{2} \xi^{2}+\ldots
$$

From (19) we obtain

$$
F^{\prime}(0)=G\left(\frac{1}{h_{2}}+\frac{1}{h_{1}}\right),
$$

where

$$
G=\frac{K^{(2)}\left(T_{0}-T_{2}\right)}{h_{2}}=\frac{K^{(1)}\left(T_{1}-T_{0}\right)}{h_{1}}
$$

is the equilibrium heat flux from the plane $y=-h_{1}$ to the plane $y=h_{2}$. Thus, the linearized interfacial condition (18) becomes

$$
\rho^{(1)}\left[\frac{\partial \Phi^{(1)}}{\partial y}-\left(\frac{\partial}{\partial t}+U^{(1)} \frac{\partial}{\partial x}\right) \xi\right]=\alpha \xi,
$$


where $\alpha=F^{\prime}(0) / L$, and $F^{\prime}(0)$ is given by (21). Substitution of (15) into (17) and (23) and solving the resulting equations to obtain $C_{j}, j=1,2$, the solutions (15) can be written in the form

$$
\begin{aligned}
\Phi^{(j)}= & \pm \frac{\xi \cosh k\left(y \pm h_{j}\right)}{k \rho_{j} \sinh k h_{j}} \\
& \cdot\left\{\alpha-i \rho_{j}\left(\omega-k U^{(j)}\right)\right\} .
\end{aligned}
$$

(3) The tangential component of the electric field must be continuous across the interface, i.e.

$$
\begin{aligned}
\frac{\partial \Psi_{1}^{(1)}}{\partial x} & -E_{0}^{(1)} \sin \theta_{1} \frac{\partial \xi}{\partial x} \\
& =\frac{\partial \Psi_{1}^{(2)}}{\partial x}-E_{0}^{(2)} \sin \theta_{2} \frac{\partial \xi}{\partial x} .
\end{aligned}
$$

(4) The normal electric displacement must be continuous across the interface, i.e.

$$
\begin{aligned}
& \varepsilon^{(1)}\left[\frac{\partial \Psi_{1}^{(1)}}{\partial y}+E_{0}^{(1)} \cos \theta_{1} \frac{\partial \xi}{\partial x}\right] \\
& =\varepsilon^{(2)}\left[\frac{\partial \Psi_{1}^{(2)}}{\partial y}+E_{0}^{(2)} \cos \theta_{2} \frac{\partial \xi}{\partial x}\right] .
\end{aligned}
$$

From (15), (25), and (26), we obtain

$$
\begin{aligned}
\Psi_{1}^{(j)}= & \frac{\xi \sinh k\left(y \pm h_{j}\right)}{\left(\varepsilon^{(2)} \tanh k h_{1}+\varepsilon^{(1)} \tanh k h_{2}\right) \cosh k h_{j}} \\
& \cdot\left[\varepsilon^{(j \pm 1)}\left(E_{0}^{(1)} \sin \theta_{1}-E_{0}^{(2)} \sin \theta_{2}\right) \pm i \tanh k h_{j \pm 1}\right. \\
& \cdot\left(\varepsilon^{(2)}\left(E_{0}^{(2)} \cos \theta_{2}-\varepsilon^{(1)} E_{0}^{(1)} \cos \theta_{1}\right] .\right.
\end{aligned}
$$

(5) Force equilibrium at the interface requires that the pressure jump across the interface balances the surface tension and electric stresses [26], i.e.

$$
\begin{aligned}
{\left[p^{(1)}-p^{(2)}\right.} & \left.+\sigma\left(\frac{1}{R_{1}}+\frac{1}{R_{2}}\right)\right] n_{i} \\
& =\left(\tau_{i k}^{(1)}-\tau_{i k}^{(2)}\right) n_{k},
\end{aligned}
$$

where $p$ is the pressure, $\sigma$ the surface tension coefficient, $R_{1}$ and $R_{2}$ are the principal radii of curvature of the interface, $\tau_{i k}=\varepsilon E^{(i)} E^{(k)}-\frac{1}{2} \varepsilon E^{2} \delta_{i k}$ is the Maxwell stress tensor, and $n_{i}, n_{k}$ are the components of the unit normal vector $\boldsymbol{n}$ to the interface in the $x$ - and $y$-directions, respectively, where

$$
\boldsymbol{n}=\frac{\nabla S}{|\nabla S|}=-\frac{\partial \xi}{\partial x} \boldsymbol{e}_{x}+\boldsymbol{e}_{y}
$$

to the first order terms, using (6).

\section{Dispersion Equation and Discussion}

Eliminating the pressure using Bernoulli's equation, then the above condition (28), upon substitution for $\Phi^{(j)}$, and $\Psi_{1}^{(j)}, j=1,2$ from (24) and (27), yields the dispersion relation

$$
\begin{aligned}
& \omega^{2}\left(\varrho^{(1)} \operatorname{coth} k h_{1}+\varrho^{(2)} \operatorname{coth} k h_{2}\right) \\
& \quad+\omega\left[i \alpha\left(\operatorname{coth} k h_{1}+\operatorname{coth} k h_{2}\right)\right. \\
& \quad-2 k\left(\varrho^{(1)} U^{(1)} \operatorname{coth} k h_{1}+\left(\varrho^{(2)} U^{(2)} \operatorname{coth} k h_{2}\right)\right] \\
& \quad-\left[g k\left(\varrho^{(1)}-\varrho^{(2)}\right)+\sigma k^{3}-k^{2}\left(\varrho^{(1)} U^{(1)^{2}} \operatorname{coth} k h_{1}\right.\right. \\
& \left.\left.\quad+\varrho^{(2)} U^{(2)^{2}} \operatorname{coth} k h_{2}\right)\right]-i \alpha k\left(U^{(1)} \operatorname{coth} k h_{1}\right. \\
& \left.\quad+U^{(2)} \operatorname{coth} k h_{2}\right)-k^{2}\left(\varepsilon^{(2)} \tanh k h_{1}\right. \\
& \left.\quad+\varepsilon^{(1)} \tanh k h_{2}\right)^{-1}\left[\left(\varepsilon^{(2)} E_{0}^{(2)} \cos \theta_{2}\right.\right. \\
& \left.\quad-\varepsilon^{(1)} E_{0}^{(1)} \cos \theta_{1}\right)^{2} \tanh k h_{1} \tanh k h_{2} \\
& \left.\quad-\varepsilon^{(1)} \varepsilon^{(2)}\left(E_{0}^{(2)} \sin \theta_{2}-E_{0}^{(1)} \sin \theta_{1}\right)^{2}\right] \\
& \quad-\left(i k^{2} / 2\right)\left(\varepsilon^{(2)} E_{0}^{(2)^{2}} \sin 2 \theta_{2}-\varepsilon^{(1)} E_{0}^{(1)^{2}} \sin 2 \theta_{1}\right)=0
\end{aligned}
$$

If liquid 1 is hotter than liquid 2, i.e. $T_{1}>T_{0}>T_{2}$, then $L$ is positive and $G$ is positive. Also, if liquid 2 is hotter than liquid 1, i.e. $T_{1}<T_{0}<T_{2}$, then $L$ and $G$ are both negative. In both cases, $\alpha$ is always positive. Note that, when $\alpha=0$, and in the absence of electric field and fluid velocities, we recover the classical Rayleigh-Taylor dispersion relation [1].

Firstly, we shall discuss the Rayleigh-Taylor instability case, i.e. when $U^{(1)}=U^{(2)}=0$. In this case the dispersion relation (30) can be written in the form

$\omega^{2}\left(\varrho^{(1)} \cosh k h_{1} \sinh k h_{2}+\varrho^{(2)} \cosh k h_{2} \sinh k h_{1}\right)$

$+i \alpha \omega \sinh k\left(h_{1}+h_{2}\right)+\left[g k\left(\varrho^{(2)}-\varrho^{(1)}\right)-\sigma k^{3}\right]$

$\times \sinh k h_{1} \sinh k h_{2}-k^{2} \sinh k h_{1} \sinh k h_{2}$

$\times\left(\varepsilon^{(2)} \tanh k h_{1}+\varepsilon^{(1)} \tanh k h_{2}\right)^{-1}\left[\left(\varepsilon^{(2)} E_{0}^{(2)} \cos \theta_{\text {; }}\right.\right.$

$\left.-\varepsilon^{(1)} E_{0}^{(1)} \cos \theta_{1}\right)^{2} \tanh k h_{1} \tanh k h_{2}$

$\left.-\varepsilon^{(1)} \varepsilon^{(2)}\left(E_{0}^{(2)} \sin \theta_{2}-E_{0}^{(1)} \sin \theta_{1}\right)^{2}\right]$

$-\left(i k^{2} / 2\right)\left(\varepsilon^{(2)} E_{0}^{(2)^{2}} \sin 2 \theta_{2}-\varepsilon^{(1)} E_{0}^{(1)^{2}} \sin 2 \theta_{2}\right)$

$\times \sinh k h_{1} \sinh k h_{2}=0$.

Let us denote

$$
\begin{aligned}
a= & \alpha \sinh k\left(h_{1}+h_{2}\right) \\
& \cdot\left[2 \left(\rho^{(1)} \cosh k h_{1} \sinh k h_{2}\right.\right. \\
& \left.\left.+\rho^{(2)} \cosh k h_{2} \sinh k h_{1}\right)\right]^{-1},
\end{aligned}
$$




$$
\begin{aligned}
b= & \sinh k h_{1} \sinh k h_{2}\left(\rho^{(1)} \cosh k h_{1} \sinh k h_{2}\right. \\
& \left.+\rho^{(2)} \cosh k h_{2} \sinh k h_{1}\right)^{-1}\left\{g k\left(\rho^{(2)}-\rho^{(1)}\right)\right. \\
& -\sigma k^{3}-k^{2}\left(\varepsilon^{(2)} \tanh k h_{1}+\varepsilon^{(1)} \tanh k h_{2}\right)^{-1} \\
& \cdot\left[\left(\varepsilon^{(2)} E_{0}^{(2)} \cos \theta_{2}-\varepsilon^{(1)} E_{0}^{(1)} \cos \theta_{1}\right)^{2}\right. \\
& \cdot \tanh k h_{1} \tanh k h_{2} \\
& \left.-\varepsilon^{(1)} \varepsilon^{(2)}\left(E_{0}^{(2)} \sin \theta_{2}-E_{0}^{(1)} \sin \theta_{1}\right)^{2}\right] \\
& \left.-\left(i k^{2} / 2\right)\left(\varepsilon^{(2)} E_{0}^{(2)^{2}} \sin 2 \theta_{2}-\varepsilon^{(1)} E_{0}^{(1)^{2}} \sin 2 \theta_{1}\right)\right\} .
\end{aligned}
$$

Thus, (31) can be rewritten as

$$
\omega=-i a \pm\left(-a^{2}-b\right)^{1 / 2} .
$$

Recalling that a factor or $\exp [i(k x-\omega t)]$ is attached to each perturbed quantity, then when $b>0$, the system is unstable since one root of $\omega$ is positive imaginary. The condition for instability in this case is

$$
\begin{aligned}
{\left[\mathcal{E}^{(1)}\right.} & \mathcal{\varepsilon}^{(2)}\left(E_{0}^{(2)} \sin \theta_{2}-E_{0}^{(1)} \sin \theta_{1}\right)^{2} \\
& -\left(\varepsilon^{(2)} E_{0}^{(2)} \cos \theta_{2}-\varepsilon^{(1)} E_{0}^{(1)} \cos \theta_{1}\right)^{2} \\
& \left.\cdot \tanh k h_{1} \tanh k h_{2}\right] \\
> & \frac{1}{k}\left(\varepsilon^{(2)} \tanh k h_{1}+\varepsilon^{(1)} \tanh k h_{2}\right) \\
& \cdot\left[g k\left(\rho^{(1)}-\rho^{(2)}\right)+\sigma k^{2}\right] .
\end{aligned}
$$

However, since $a>0$, the growth rate of the instability is reduced from that of the classical case when $a=0$. When $b<0$, the system is stable, and the condition for stability in this case is similar to (35) when the inequality sign (>) is replaced by the sign $(<)$. Note that, for this stable system, in contrast to the classical case, there is no permanent periodicwave state, and the system will settle down to an asymptotic equilibrium because of the evaporation effect. In most physical situations, the quantity $k h_{2}$ and the ratio $\rho^{(2)} / \rho^{(1)}$ are very large; then the expres- sions of $a$ and $b$ are simplified to

$$
\begin{aligned}
a= & \frac{\alpha}{2 \rho^{(2)}}\left(1+\operatorname{coth} k h_{1}\right), \\
b= & g k\left[1-\frac{\alpha k^{2}}{g \rho^{(2)}}\right] \\
& -\frac{k^{2}}{\rho^{(2)}\left(\varepsilon^{(2)} \tanh k h_{1}+\varepsilon^{(1)}\right)}\left[\left(\varepsilon^{(2)} E_{0}^{(2)} \cos \theta_{2}\right.\right. \\
& \left.-\varepsilon^{(1)} E_{0}^{(1)} \cos \theta_{1}\right)^{2} \tanh k h_{1} \\
& \left.-\varepsilon^{(1)} \varepsilon^{(2)}\left(E_{0}^{(2)} \sin \theta_{2}-E_{0}^{(1)} \sin \theta_{1}\right)^{2}\right] \\
& -\frac{i k^{2}}{2 \rho^{(2)}}\left(\varepsilon^{(2)} E_{0}^{(2)^{2}} \sin 2 \theta_{2}-\varepsilon^{(1)} E_{0}^{(1)^{2}} \sin 2 \theta_{1}\right) .
\end{aligned}
$$

Although a direct comparison between the present result and the results from the previous more general formulation is not easy to make, it may be seen that the present result, in the absence of electric field, agrees with some of the limiting cases from the general result as discussed in the previous works of Hsieh [27], and Dhir and Lienhard [28]. It is noteworthy that the effects of heat and mass transfer are revealed through a single parameter $\alpha$, in this simplified problem. It would be interesting to see whether experimental data can indeed be correlated by this parameter.

Secondly, we shall discuss the Kelvin-Helmholtz instability case, i.e. when the fluid velocities $U^{(1)}$ and $U^{(2)}$ are included in the analysis. It is clear that when $\alpha=0$, and in the absence of the electric field, the dispersion relation (30) is reduced to that of the classical Kelvin-Helmholtz problem. When $U^{(1)}=U^{(2)}=U$, the expression for $\omega$ is the same as that of the Rayleigh-Taylor problem, except for an additive term $k U$ to take care of the streaming of the fluid. Thus when $\left|U^{(1)}-U^{(2)}\right|$ is small, the behaviour of the flow system differs little from the Rayleigh-Taylor case.

In the presence of fluid velocities, (30) can be rewritten in the form

$$
\begin{aligned}
& \omega^{2}\left(v^{(1)}+v^{(2)}\right)+\omega\left[i \alpha\left(\frac{v^{(1)}}{\rho^{(1)}}+\frac{v^{(2)}}{\rho^{(2)}}\right)-2 k\left(v^{(1)} U^{(1)}+v^{(2)} U^{(2)}\right)\right] \\
&- {\left[g k\left(\rho^{(1)}-\rho^{(2)}\right)+\sigma k^{3}\right] \sinh k h_{1} \sinh k h_{2}+k^{2}\left(v^{(1)} U^{(1)^{2}}+v^{(2)} U^{(2)^{2}}\right) } \\
&-i \alpha k\left(\frac{v^{(1)} U^{(1)}}{\rho^{(1)}}+\frac{v^{(2)} U^{(2)}}{\rho^{(2)}}\right)-\frac{k^{2} \sinh k h_{1} \sinh k h_{2}}{\left(\varepsilon^{(2)} \operatorname{coth} k h_{2}+\varepsilon^{(1)} \operatorname{coth} k h_{1}\right)} \\
& \times\left[\left(\varepsilon^{(2)} E_{0}^{(2)} \cos \theta_{2}-\varepsilon^{(1)} E_{0}^{(1)} \cos \theta_{1}\right)^{2}-\varepsilon^{(1)} \varepsilon^{(2)}\left(E_{0}^{(2)} \sin \theta_{2}\right.\right. \\
&\left.\left.-E_{0}^{(1)} \sin \theta_{1}\right)^{2} \operatorname{coth} k h_{1} \operatorname{coth} k h_{2}\right] \\
&-\left(i k^{2} / 2\right)\left(\varepsilon^{(2)} E_{0}^{(2)^{2}} \sin 2 \theta_{2}-\varepsilon^{(1)} E_{0}^{(1)^{2}} \sin 2 \theta_{1}\right) \sinh k h_{1} \sinh k h_{2}=0
\end{aligned}
$$


where,

$$
v^{(1)}=\rho^{(1)} \cosh k h_{1} \sinh k h_{2} \text { and } v^{(2)}=\rho^{(2)} \cosh k h_{2} \sinh k h_{1}
$$

The stability criterion may be determined by the condition that $\omega$ be real. Thus we obtain from (38) the critical condition

$$
\begin{aligned}
J= & \left(v^{(1)}+v^{(2)}\right)\left\{g k\left(\rho^{(1)}-\rho^{(2)}\right)+\sigma k^{3}+k^{2}\left(\varepsilon^{(2)} \operatorname{coth} k h_{2}+\varepsilon^{(1)} \operatorname{coth} k h_{1}\right)^{-1}\right. \\
& \times\left[\left(\varepsilon^{(2)} E_{0}^{(2)} \cos \theta_{2}-\varepsilon^{(1)} E_{0}^{(1)} \cos \theta_{1}\right)^{2}-\varepsilon^{(1)} \varepsilon^{(2)}\left(E_{0}^{(2)} \sin \theta_{2}-E_{0}^{(1)} \sin \theta_{1}\right)^{2}\right. \\
& \left.\left.\times \operatorname{coth} k h_{1} \operatorname{coth} k h_{2}\right]\right\} \sinh k h_{1} \sinh k h_{2} \\
& -k^{2} v^{(1)} v^{(2)}\left(U^{(1)}-U^{(1)}\right)^{2}\left[1+\left(\frac{\rho^{(2)}-\rho^{(1)}}{\rho^{(2)} v^{(1)}+\rho^{(1)} v^{(2)}}\right)^{2} v^{(1)} v^{(2)}\right]=0
\end{aligned}
$$

with

$$
\varepsilon^{(2)} E_{0}^{(2)^{2}} \sin 2 \theta_{2}=\varepsilon^{(1)} E_{0}^{(1)^{2}} \sin 2 \theta_{1} .
$$

The system is stable if $J \geq 0$, i.e. when the condition

$$
\begin{aligned}
\frac{g}{k} & \left(\rho^{(1)}-\rho^{(2)}\right)+\sigma k+\frac{1}{\left(\varepsilon^{(2)} \operatorname{coth} k h_{2}+\varepsilon^{(1)} \operatorname{coth} k h_{1}\right)}\left[\left(\varepsilon^{(2)} E_{0}^{(2)} \cos \theta_{2}\right.\right. \\
& \left.\left.-\varepsilon^{(1)} E_{0}^{(1)} \cos \theta_{1}\right)^{2}-\varepsilon^{(1)} \varepsilon^{(2)}\left(E_{0}^{(2)} \sin \theta_{2}-E_{0}^{(1)} \sin \theta_{1}\right)^{2} \operatorname{coth} k h_{1} \operatorname{coth} k h_{2}\right] \\
& \geq \frac{v^{(1)} v^{(2)}\left(U^{(1)}-U^{(2)}\right)^{2}}{\left(v^{(1)}+v^{(2)}\right) \sinh k h_{1} \sinh k h_{2}}\left[1+v^{(1)} v^{(2)}\left(\frac{\rho^{(2)}-\rho^{(1)}}{\rho^{(2)} v^{(1)}+\rho^{(1)} v^{(2)}}\right)\right]
\end{aligned}
$$

together with (41) is satisfied, and it is otherwise unstable.

It is clear from the inequality (42) that the oblique electric field has a stabilizing effect on the considered system, and this stability is reduced by increasing the normal electric field components. This result coincides with the well-known result of the linear electrohydrodynamic stability which states that the normal electric field has always a destabilizing influence on the configuration [17]. It is clear also that the stability increases when $0<\theta_{1}, \theta_{2}<\pi / 4$, and decreases when $\pi / 4<\theta_{1}$, $\theta_{2}<\pi / 2$, while the instability increases for large values of the depths $h_{1}$ and $h_{2}$. In our case of applied electric fields we note that the new condition (41) should also be satisfied for stability. The case when $\theta_{1}=\theta_{2}=0$ corresponds to tangential electric fields, showing a stabilizing effect of the electric field, and in this case the stability criterion is governed by (42) only. Also the case when $\theta_{1}=\theta_{2}=\pi / 2$ corresponds to normal electric fields, showing thereby a destabilizing influence of the electric field as shown by (42). In both cases the condition (41) is automatically satisfied, and it does not affect the stability criterion. If $\theta_{1}=\theta_{2}=\pi / 4$, then the system will be stable as well as unstable depending on the electric fields and dielectric constants related by the equation $\varepsilon^{(2)} E_{0}^{(2)^{2}}=\varepsilon^{(1)} E_{0}^{(1)^{2}}$. Finally, note that in the absense of the oblique electric fields, (40) reduces to the equation obtained earlier by Hsieh [2] for the pure hydrodynamical case in the presence of heat and mass transfer, and therefore his results are recovered. Note also that the critical wavenumber above which the system will be stable can be determined from condition (42) only for some physical problems of interest whem $k h_{1}, k h_{2}$ are very small or very large individually or together.

The expression for $J$, in the presence of an electric field, differs from the classical Kelvin-Helmholtz problem by the additional last term. It is somewhat surprizing that the parameter $\alpha$ does not appear in the expression. Thus, this expression is valid even for infinitesimal $\alpha$, and yet when $\alpha=0$, the last term is absent. There is no anomaly, however, if we look at the growth rate of the instability. When $\alpha$ is infinitesimally small, the additional effect on the growth rate of the instability is also infinitesimally small.

The discrepancy can also be seen from an other angle. The vanishing of the imaginary part of the dispersion relation determines the wave speed for the critical case, the equation

$$
\frac{\omega}{k}=\frac{\left(\rho^{(2)} v^{(1)} U^{(1)}+\rho^{(1)} v^{(2)} U^{(2)}\right)}{\left(\rho^{(2)} v^{(1)}+\rho^{(1)} v^{(2)}\right)}
$$


together with condition (41). In contrast, the critical wave speed for the classical Kelvin-Helmholtz instability is

$$
\frac{\omega}{k}=\frac{\left(v^{(1)} U^{(1)}+v^{(2)} U^{(2)}\right)}{\left(v^{(1)}-v^{(2)}\right)} .
$$

To summarize, we have presented a novel mathematical formulation for the effect of oblique electric fields on the interfacial stability problems with heat and mass transfer. For the case of the Rayleigh-Taylor instability problem, it is found that the simplified problem retains the essential feature that, although the effect of heat and mass transfer tends to reduce the growth rate of the instability, the criterion for stability is still the same as the classical result. For this formulation, the effects of heat and mass transfer are revealed through one single parameter $\alpha$. Thus, correlation of experimental data would greatly facilitated by this simplification. For such physical problems as film boiling, nonlinear effects are

[1] S. Chandrasekhar, Hydrodynamic and Hydromagnetic Stability, Oxford University Press, Oxford 1961.

[2] D.-Y. Hsieh, Phys. Fluids 21, 745 (1978).

[3] A. R. Nayak and B. B. Chakraborty, Phys. Fluids 27, 1937 (1984).

[4] S.-P. Ho, J. Fluid Mech. 101, 111 (1980).

[5] L. A. Mel'nikovskii and S. A. Kriminskii, Sov. Phys. JETP 84, 758 (1997)

[6] K. Adham-Khodaparast, M. Kawaji, and B. N. Antar, Phys. Fluids 7, 359 (1995).

[7] G. M. Moatimid and Y. O. El-Dib, Int. J. Theor. Phys. 35, 425 (1996).

[8] G. M. Moatimid, Int. J. Enging. Sci. 32, 535 (1994).

[9] N. T. Eldabe, J. Fac. Education (Egypt) 13, 303 (1988).

[10] D.-Y. Hsieh, Phys. Fluids 22, 1435 (1979).

[11] R. K. Chhabra and S. K. Trehan, Indian J. Pure Appl. Math. 25, 541 (1994)

[12] A. R. F. Elhefnawy, Y. O. El-Dib, and Y. D. Mahmoud, Int. J. Theor. Phys. 36, 2079 (1997).

[13] A. R. F. Elhefnawy, Arab. J. Sci. Enging. 21, 129 (1996).

[14] A. A. Mohamed, A. R. F. Elhefnawy, and Y. D. Mahmoud, Physica A 195, 74 (1993); 202, 264 (1994); J. Phys. Soc. Japan 64, 4693 (1995).

[15] M. H. Obied-Allah and A. A. Yahia, Astrophys. Space Sci. 181, 183 (1991).

[16] D.-Y. Hsieh and S.-P. Ho, Phys. Fluids 24, 202 (1981); D.-Y. Hsieh and F. Chen, Phys. Fluids 28, 1253 (1985). essential. Then, the simplified formulation is even more valuable for the difficult analysis of nonlinear stability. The study of nonlinear hydromagnetic Rayleigh-Taylor, and Kelvin-Helmholtz instability problems with heat and mass transfer will be reported in subsequent papers. For the case of the present Kelvin-Helmholtz problem, the new stability criterion raises some questions which perhaps can only be answered by a detailed analysis from a more comprehensive formulation of the problem, and this will be done in the near future.

\section{Acknowledgements}

The financial support of Natural Sciences and Engineering Research Council of United Arab Emirates University is gratefully acknowledged. I would like also to thank Prof. Dr. D. K. Callebaut (University of Antwerp, Belgium) for his critical reading of the manuscript, and his useful comments.

[17] J.-R. Melcher, Continuum Electromechanics, MIT Press, Cambridge, Mass 1981.

[18] R. J. Turnbull, Phys. Fluids 11, 2588 (1968).

[19] H. Y. Choi, Electrohydrodynamic Boiling Heat Transfer, Ph. D. Thesis, MIT, Cambridge (Mass) 1962.

[20] E. Bonjour, J. Verdier, and L. Weil, Chem. Enging. Progress 58, 63 (1962).

[21] R. L. Johnson, AIAA J. 6, 1456 (1968).

[22] M. Markels and R. L. Durfee, AIChE J. 10, 106 (1964); 11, 716 (1965)

[23] T. B. Jones and R. C. Schaeffer, AIAA J. 14, 1759 (1976).

[24] M. F. El-Sayed, Physica Scripta 55, 350 (1997); 58, 613 (1998); Can. J. Phys. 75, 499 (1997); Physica A 255, 1 (1998); Z. Naturforsch. A 53, 17 (1998); 54 (1999) (in press); Nuovo Cim. D 20, 1645 (1998); Czeck. J. Phys. 49, 473 (1999).

[25] M. F. El-Sayed and D. K. Callebaut, Physica Scripta 57, 161 (1998); J. Colloid Interface Sci. 200, 203 (1998); Z. Naturforsch. A 53, 217 (1998); Physica A (1999) (in press).

[26] L. D. Landau and E. M. Lifshitz, Fluid Mechanics, Pergamon Press, Oxford 1987; Electrodynamics of Continuous Media, Pergamon Press, Oxford 1960.

[27] D.-Y. Hsieh, Trans. ASME D 49, 156 (1972).

[28] V. Dhir and J. Lienhard, Trans ASME D 49, 160 (1972). 